\title{
FUNCTIONS TYPICALLY-REAL AND MEROMORPHIC IN THE UNIT CIRCLE
}

BY

A. W. GOODMAN

1. Introduction. In $1932 \mathrm{~W}$. Rogosinski [15] introduced and studied quite thoroughly the class of functions typically-real in the unit circle, i.e., functions of the form

$$
f(z)=z+\sum_{n=2}^{\infty} b_{n} z^{n}
$$

which are regular in $|z|<1$ (hereafter denoted by $E$ ) and in addition satisfy in $E$ the condition

$$
\Im f(z) \Im z \geqq 0 .
$$

This concept has been extended in several directions in $[4 ; 6 ; 10 ; 11 ; 13 ; 16]$. In the present paper we initiate the study of functions which are meromorphic in $E$ but still satisfy the condition (1.2). To be precise we make the following definitions.

A function $f(z)$ which is meromorphic in $E$, and except at the poles satisfies the condition (1.2), is said to be meromorphic and typically-real in the unit circle. The class of such functions with Taylor series of the form (1.1) in a neighborhood of the origin will be denoted by TM, and the subclass of functions regular in $E$ will be denoted by TR. The class of functions meromorphic and typically-real in $E$ with a Laurent expansion about the origin of the form

$$
\phi(z)=-1 / z+\sum_{n=0}^{\infty} \beta_{n} z^{n}
$$

will be denoted by TM*.

It is immediately obvious that the condition (1.2) implies that, for a function meromorphic and typically-real in $E$, all the poles lie on the interval $-1<z<1$, are of first order, and have negative residues. Hence, by a trivial transformation any such function can be reduced to either the form (1.1) or (1.3), and in either case all of the coefficients are real. The set of functions TM is convex, for if $f_{1}(z)$ and $f_{2}(z)$ belong to TM, so also does

$$
f(z)=m_{1} f_{1}(z)+m_{2} f_{2}(z)
$$

for every pair $m_{1}, m_{2}>0$, with $m_{1}+m_{2}=1$. A similar remark can be made for

Presented to the Society, December 29, 1954; received by the editors January 17, 1955. 
the set $\mathrm{TM}^{*}$. We also note an elementary relation between the two sets, namely if $f(z) \in \mathrm{TM}$, then $\phi(z)=-f(z)^{-1} \in \mathrm{TM}^{*}$ and conversely.

Among other results, we determine, for functions of these two classes, the precise domain of variability of $f(z)$ and $\phi(z)$, and sharp bounds for the coefficients $b_{n}$ and $\beta_{n}$ in terms of the modulus of the closest pole to the origin.

2. Bounds for functions belonging to TM. We first prove the following theorem.

TheOREM 1. Let $f(z) \in T M$. Then for each nonreal $a=A e^{i \alpha}$ in $E$

$$
\left|\frac{f^{\prime}(a)}{\Im f(a)}\right| \leqq \frac{\left|1-a^{2}\right|}{A\left(1-A^{2}\right)|\sin \alpha|} \equiv M_{1}(a)
$$

and

$$
\left|\frac{f^{\prime}(a)}{f(a)}\right| \leqq M_{1}(a),
$$

and these bounds are sharp. The equality sign occurs in (2.1) if and only if

$$
f(z)=F_{s}(z)=\frac{z}{1-2 s z+z^{2}},
$$

s real.

The equality sign occurs in (2.2) if and only if for $z=a, \Re F_{s}(a)=0$, that is for the function (2.3) with

$$
2 s=\left(A^{-1}+A\right) \cos \alpha .
$$

We remark that the form of the left side of (2.1) is a little unusual, and that the extremal function is not unique, since equality occurs for every real s. Clearly (2.2) is a trivial consequence of (2.1). We also note that because $f(z)$ is real on the real axis it is sufficient to consider only the upper half of $E$, i.e., we may assume $\sin \alpha>0$, and this assumption is made throughout this paper.

To prove this theorem let $z=g(\zeta)$ map $|\zeta|<1$ conformally onto the semicircular region $\Im z>0,|z|<1$, with $g(0)=a$. The inverse function $\zeta=G(z)$ is a rational function and indeed has the form

$$
G(z)=e^{i \gamma} \frac{(z-a)(1-a z)}{(z-\bar{a})(1-\bar{a} z)},
$$

$\gamma$ real.

If now $f(z) \in T M$, the function

$$
f_{1}(\zeta)=-i f(g(\zeta))=\sum_{n=0}^{\infty} A_{n} \zeta^{n}
$$

is regular and $\Re f_{1}(\zeta)>0$, for $|\zeta|<1$. By a fundamental theorem of Carathéodory $[1 ; 2]$, 
with equality if and only if $f_{1}(\zeta)$ maps $|\zeta|<1$ onto the right half-plane. An easy computation gives

$$
\left|A_{1}\right|=\left|f^{\prime}(a) g^{\prime}(0)\right|=\left|f^{\prime}(a)\right| 2 M_{1}^{-1}(a) \leqq 2 \Re A_{0}=2 \Im f(a),
$$

and hence (2.1). For equality in (2.1), $f(z)$ must map the semicircular region onto the upper half plane. Since $f(z) \in T M$ it must then map $E$ onto the full plane minus a set on the real axis. This set may be either a segment, an infinite ray, or two infinite rays. But all such functions have the form (2.3). Conversely, an easy computation shows that for $F_{s}(z)$, the two sides of $(2.1)$ are identical for every $a$, independent of $s$.

Obviously other bounds can be obtained from (2.7) using $n \geqq 2$, but these are more complicated than (2.1) and do not seem to be fruitful. On the other hand, (2.1) leads quite naturally to the following result.

THEOREM 2. With the conditions and notations of Theorem 1 ,

$$
|f(a)| \leqq \frac{A}{\left(1-A^{2}\right)|\sin \alpha|} \equiv M_{2}(a),
$$

and this bound is sharp. The equality sign occurs only for $F_{s}(z)$ given by (2.3) and (2.4).

Proof. If $f(z) \in \mathrm{TM}$ then $-f(-z) \in \mathrm{TM}$, so without loss of generality we assume $a$ is in the first quadrant, but not real. Let $\Gamma$ be the portion of the curve

$$
2 s=(r+1 / r) \cos \theta,
$$$$
z=r e^{i \theta},
$$

joining $z=0$ with $z=a$, where $s$ is defined by (2.4), and let $a_{0}=A_{0} e^{i \alpha}$ be a point on $\Gamma$ between $z=0$ and $z=a$. Integrating both sides of the inequality (2.2) on $\Gamma$ from $a_{0}$ to $a$ yields

$$
\left|\log \frac{f(a)}{f\left(a_{0}\right)}\right|=\left|\int_{a_{0}}^{a_{0}} \frac{f^{\prime}(z)}{f(z)} d z\right| \leqq \int_{a_{0}}^{a} M_{1}(z) d \sigma \equiv I
$$

where $\sigma$ is the length of arc on $\Gamma$. A brief computation shows that with $z=r e^{i \theta}$, and with $\theta$ regarded as a function of $r$ on $\Gamma$,

$$
d \sigma=\frac{\left(1-2 r^{2} \cos 2 \theta+r^{4}\right)^{1 / 2}}{\left(1+r^{2}\right) \sin \theta} d r
$$

while

$$
M_{1}(z)=\frac{\left(1-2 r^{2} \cos 2 \theta+r^{4}\right)^{1 / 2}}{r\left(1-r^{2}\right) \sin \theta}
$$


Thus we can write for $I$,

$$
I=\int_{A_{0}}^{A}\left\{\frac{1-r^{2}}{r\left(1+r^{2}\right) \sin ^{2} \theta}+\frac{4 r}{1-r^{4}}\right\} d r,
$$

and using (2.10) to eliminate $\theta$,

$$
I=\int_{A_{0}}^{A}\left\{\frac{1-r^{4}}{r\left[\left(1+r^{2}\right)^{2}-4 s^{2} r^{2}\right]}+\frac{4 r}{1-r^{4}}\right\} d r .
$$

On the other hand, on the curve $\Gamma$,

$$
M_{2}(z)=\frac{r}{\left(1-r^{2}\right) \sin \theta}=\frac{r\left(1+r^{2}\right)}{\left(1-r^{2}\right)\left\{\left(1+r^{2}\right)^{2}-4 r^{2} s^{2}\right\}^{1 / 2}}
$$

and $d \log M_{2}(z) / d r$ is just the integrand of (2.15). Thus (2.11) yields

$$
\left|\log \frac{f(a)}{f\left(a_{0}\right)}\right| \leqq \log \frac{M_{2}(a)}{M_{2}\left(a_{0}\right)}
$$

with equality only if $f(z)$ is given by (2.3) and (2.4). An easy manipulation of (2.17) and passage to the limit as $a_{0} \rightarrow 0$ gives (2.9). The bounds obtained in Theorems 1 and 2 give immediately the bound for $f^{\prime}(z)$.

THEOREM 3. With the conditions and notations of Theorem 1

$$
\left|f^{\prime}(a)\right| \leqq \frac{\left|1-a^{2}\right|}{\left(1-A^{2}\right)^{2} \sin ^{2} \alpha}
$$

and this bound is sharp. The equality sign occurs only for $F_{s}(z)$ given by (2.3) and (2.4).

3. The domain of variability for $f(z)$ in TM. Let $z=a$ be a fixed point of $E$, then the set $D$ of all points $f(a)$ where $f(z) \in$ TM is called the domain of variability of $f(z)$ at $a$. If $w$ is a point of $D$ and $f(z) \in \mathrm{TM}$ is such that $f(a)=w$, we then say that $f(z)$ corresponds to the point $w$ of $D$. From equation (1.4) it is obvious that for each $a, D$ is convex. We shall see that if $a$ is not real, $D$ is a circle.

Theorem 4. Let $a=A e^{i \alpha}$ be $a$ point of $E$ with $\sin \alpha>0$, and let $K(a)$ be the circular disk

$$
\left|w-i \frac{M_{2}(a)}{2}\right| \leqq \frac{M_{2}(a)}{2},
$$

minus the point $w=0$. Then $K(a)$ is the domain of variability for $f(z) \in \mathrm{TM}$ at $z=a$. Further to each $w \neq 0$ on the boundary of $K(a)$ there corresponds exactly one function of TM, and this is of the form (2.3). To each interior point of $K(a)$ there correspond infinitely many functions. 
Proof. If $c$ is a real constant, the parabolic transformation [3, p. 23]

$$
w_{1}=\frac{w}{1+c w}=w-c w^{2}+\cdots
$$

takes each circular disk $K(a)$ into itself, the interior going into the interior and the boundary going into the boundary. Further, (3.2) preserves the upper half-plane and the lower half-plane so that if $w=f(z) \in \mathrm{TM}$, so also is $w_{1}=w_{1}(z)$. Assume now that for a fixed $a, f(a)$ does not lie in $K(a)$. Then by an appropriate choice of $c$ in (3.2), $w_{1}(a)$ can be made purely imaginary. Since $w_{1}(a)$ is also not in $K(a)$ it follows that $\left|w_{1}(a)\right|>M_{2}(a)$, a contradiction to Theorem 2. If $f(a)$ is on the boundary of $K(a)$, then with proper choice of $c$, $w_{1}(a)=i M_{2}(a)$ and in this case the function $w_{1}(z)$ is uniquely determined. But the class of functions $F_{s}(z)$ given by $(2.3)$ is invariant under the group of transformations (3.2) so $f(z)$ must be a uniquely determined function of the form (2.3).

To see that every interior point $w_{0}$ of $K(a)$ corresponds to infinitely many functions, note first that any such point is an interior point of infinitely many chords of the circle and hence can be expressed in infinitely many ways in the form $w_{0}=m_{1} w_{1}+m_{2} w_{2}$ where $m_{1}+m_{2}=1, m_{1}>0, m_{2}>0$, and $w_{1}$ and $w_{2}$ are points on the boundary of $K(a)$. If $F_{s_{1}}(z)$ and $F_{s_{2}}(z)$ correspond to $w_{1}$ and $w_{2}$ at $a$, then (1.4) with these functions defines an $f(z) \in \mathrm{TM}$ which corresponds to $w_{0}$. That these functions are different for two different chords follows from the fact that the poles of $F_{s}(z)$ are different for different values of $s$. The case $\sin \alpha<0$ is trivial from the symmetry of the mapping.

If $\phi(z) \in \mathrm{TM}^{*}$, then $-\phi(z)^{-1} \in \mathrm{TM}$. This gives immediately the following result.

Corollary 1. Let $a=A e^{i \alpha}$ be a point of $E$ with $\sin \alpha>0$. The domain of variability for $\phi(z) \in \mathrm{TM}^{*}$ at $z=a$ is the half-plane $\Im_{w} \geqq\left(A^{-1}-A\right) \sin \alpha$. The points on the boundary correspond to the unique function $\phi(z)=-z^{-1}+2 s-z$ with appropriate real s.

The proof of Theorem 1 can be carried over without alteration to functions $\phi(z)$ of $\mathrm{TM}^{*}$. Only the extremal function is different. This gives immediately the following result.

TheOREM 5. Let $\phi(z) \in \mathrm{TM}^{*}$. Then for each nonreal $a$ in $E$

$$
\left|\frac{\phi^{\prime}(a)}{\Im \phi(a)}\right| \leqq M_{1}(a)
$$

with equality if and only if $\phi(z)=-z^{-1}-z+2 s, s$ real.

It should be observed that Theorem 5 does not follow from Theorem 1 in the same way that Corollary 1 follows from Theorem 4 . Indeed, that process would yield only $\left|\phi^{\prime}(a) / \phi(a)\right| \leqq M_{:}(a)$, which is weaker than (3.3). 
4. Bounds for the coefficients. It is clear that if the function $F_{s}(z)$ given by (2.3) is expanded about the origin, the coefficients may be made arbitrarily large by letting $s \rightarrow \infty$, i.e., by letting one pole tend to the origin. If, however, we bound the poles of $f(z)$ away from the origin, then sharp upper and lower bounds can be obtained for the coefficients. Let $f(z) \in \mathrm{TM}$, and number its poles (if any) $p_{j}$ so that

$$
0<\left|p_{1}\right| \leqq\left|p_{2}\right| \leqq \cdots \leqq\left|p_{k}\right| \leqq\left|p_{k+1}\right| \leqq \cdots<1 .
$$

If the function has only a finite number of poles, (4.1) contains only a finite number of terms, but it is clear that the class TM contains functions with infinitely many poles. The equality sign can occur in (4.1) if $p_{k}=-p_{k+1}$, but for every $k,\left|p_{k}\right|<\left|p_{k+2}\right|$. If $\left|p_{k}\right|<\left|p_{k+1}\right|$, let $R_{k}$ denote the region $\left|p_{k}\right|$ $<|z|<\left|p_{k+1}\right|$. Then there is either an $R_{k}$ with outer boundary $|z|=1$, or an infinite sequence of $R_{k}$ such that the outer boundary tends to $|z|=1$.

Theorem 6. Let $f(z) \in \mathrm{TM}$ and let the residue at the pole $p_{j}$ be $-m_{j}, m_{j}>0$ $(j=1,2, \cdots)$. Then for each $r,\left|p_{k}\right|<r<\left|p_{k+1}\right|$,

$$
\begin{aligned}
& \sum_{j=1}^{k} m_{j}\left(\frac{r^{2}}{p_{j}^{2}}-1\right) \leqq r^{2}, \\
& \sum_{j=1}^{\infty} m_{j}\left(\frac{1}{p_{j}^{2}}-1\right) \leqq 1,
\end{aligned}
$$

and

$$
0<m_{j} \leqq \frac{p_{i}^{2}}{1-p_{i}^{2}}, \quad j=1,2, \cdots .
$$

Further, the inequalities (4.3) and (4.4) are sharp. Given any sequence of positive numbers $\left\{m_{j}\right\}$ and any sequence of poles $\left\{p_{j}\right\}$ on the interval $-1<z<1$ such that (4.3) with the equality sign is satisfied, there is a function $f(z) \in \mathrm{TM}$ with the prescribed poles $p_{j}$ and residues $-m_{j}$. Equality can occur on the right of (4.4) only if $f(z)$ has just one pole.

Proof. Let the Laurent series for $f(z)$ in the ring $R_{k}$ be

$$
f(z)=\sum_{n=-\infty}^{+\infty} b_{n}^{(k)} z^{n}, \quad k=1,2,3, \cdots,
$$

and in the neighborhood of the origin let $f(z)$ be given by (1.1). We will obtain useful relationships among the coefficients. If $\left|p_{k-1}\right|<\left|p_{k}\right|<\left|p_{k+1}\right|$ then the function $g(z)=f(z)+m_{k} /\left(z-p_{k}\right)$ is regular in $\left|p_{k-1}\right|<|z|<\left|p_{k+1}\right|$. If we set

$$
g(z)=\sum_{n=-\infty}^{+\infty} b_{n}^{*} z^{n}
$$


in this region, we find immediately that in $R_{k}$

$$
f(z)=\sum_{n=-\infty}^{+\infty} b_{n}^{*{ }^{n}}-\sum_{n=1}^{\infty} m_{k} p_{k}^{n-1} \frac{1}{z^{n}},
$$

while in $R_{k-1}$

$$
f(z)=\sum_{-\infty}^{+\infty} b_{n}^{* n}+\sum_{n=0}^{\infty} \frac{m_{k}}{p_{k}^{n+1}} z^{n} .
$$

From (4.5), (4.7), and (4.8) it follows that

$$
\begin{aligned}
& b_{n}^{(k)}=b_{n}^{*}, \\
& b_{n}^{(k-1)}=b_{n}^{*}+\frac{m_{k}}{p_{k}^{n+1}} \quad \text { for } n \geqq 0, \\
& b_{n}^{(k)}=b_{n}^{*}-\frac{m_{k}}{p_{k}^{n+1}}, \quad b_{n}^{(k-1)}=b_{n}^{*}, \quad \text { for } n<0 .
\end{aligned}
$$

These lead to

$$
b_{n}^{(k)}=b_{n}^{(k-1)}-\frac{m_{k}}{p_{k}^{n+1}}, \quad n=0, \pm 1, \pm 2, \cdots .
$$

In the special case that $\left|p_{k-1}\right|=\left|p_{k}\right|$, a trivial modification shows that

$$
b_{n}^{(k)}=b_{n}^{(k-2)}-\frac{m_{k}}{p_{k}^{n+1}}-\frac{m_{k-1}}{p_{k-1}^{n+\downarrow}}, \quad n=0 \pm 1, \pm 2, \cdots,
$$

and iteration of (4.10) and (4.11) yields

$$
b_{n}^{(k)}=b_{n}-\sum_{j=1}^{k} \frac{m_{j}}{p_{i}^{n+1}}, \quad n=0, \pm 1, \pm 2, \cdots,
$$

where $b_{n}=0$ for $n \leqq 0$.

Now with the usual $z=r e^{i \theta}, v(r, \theta)=\Im f(z)$ we have for $\left|p_{k}\right|<r<\left|p_{k+1}\right|$, and for $f(z) \in \mathrm{TM}$,

$$
0 \leqq \int_{0}^{2 \pi} v(r, \theta) \sin \theta d \theta=\left(b_{1}^{(k)} r-b_{-1}^{(k)} r^{-1}\right) .
$$

This, together with (4.12) and the normalization $b_{1}=1$, yields (4.2). Taking $r$ on a suitable sequence tending to 1 yields (4.3). Using just one term from the right side of (4.3) yields (4.4).

To show that the inequality (4.3) is sharp in all the variables, suppose a sequence $\left\{p_{j}\right\}$ of poles and a sequence $\left\{-m_{j}\right\}$ of negative residues are given, such that (4.3) with the equality sign holds. Then define $F_{s j}$ by (2.3) with $2 s_{j}=p_{j}+p_{j}^{-1}(j=1,2, \cdots)$, and let 


$$
F(z)=\sum_{j=1}^{\infty} m_{j} \frac{1-p_{j}^{2}}{p_{j}^{2}} F_{s j}(z)=z+\cdots .
$$

Clearly $F(z) \in \mathrm{TM}$, and an easy computation shows that the poles and residues are as prescribed, and (4.3) holds with the equality sign.

THEOREM 7. Let $T M(p)$ denote the subclass of $T M$ of functions for which the poles $p_{j}$ satisfy $\left|p_{j}\right| \geqq p>0, j=1,2,3, \cdots$ Let

$$
B(n, p)=\left(1+p^{2}+p^{4}+\cdots+p^{2 n-2}\right) / p^{n-1}
$$

and let

$$
\mu(n)=\min _{0 \leqq \theta \leqq 2 \pi} \frac{\sin n \theta}{\sin \theta}<0, \quad n=2,3, \cdots .
$$

If $f(z) \in T M(p)$ has the form (1.1) near the origin then

$$
\begin{aligned}
-B(n, p) & \leqq b_{n} \leqq B(n, p), \\
\mu(n) & \leqq b_{n} \leqq B(n, p),
\end{aligned}
$$

$n$ even, $n$ odd,

and all these inequalities are sharp.

It is interesting that the lower bound in (4.18) is identical with that found by Rogosinski [15] in the case $f(z)$ regular, while the other three bounds are different. If $p=1$, then $\operatorname{TM}(p)$ is the class $T R$, while on the other hand, $B(n, 1)=n$. Thus Theorem 7 contains the result of Rogosinski as a special case.

Proof. Following Nehari and Schwarz [11] we note that if $f(z) \in T M$ and $\left|p_{k}\right|<|z|=r<\left|p_{k+1}\right|$, then for $n=1,2,3, \cdots$,

$$
0 \leqq \int_{0}^{2 \pi} v(r, \theta) \sin \theta(1-\cos n \theta) d \theta
$$

or

$$
0 \leqq 2 b_{1}^{(k)} r-2 b_{-1}^{(k)} r^{-1}-b_{n+1}^{(k)} r^{n+1}+b_{n-1}^{(k)} r^{n-1}+b_{-n-1}^{(k)} r^{-n-1}-b_{-n+1}^{(k)} r^{-n+1} .
$$

Using (4.12) and a little manipulation gives

$$
b_{n+1} \leqq \frac{2}{r^{n}}+\frac{b_{n-1}}{r^{2}}+\frac{1}{r^{n+2}} \sum_{j=1}^{k} m_{i}\left(\frac{r^{2}}{p_{i}^{2}}-1\right)\left(\frac{r^{n}}{p_{j}^{n}}-2+\frac{p_{j}^{n}}{r^{n}}\right) .
$$

Now $y-2+y^{-1} \geqq 0$ for $y>1$ and is increasing with $y$. Thus for fixed $p>0$ the last factor in (4.20) is a maximum if $p_{j}=p(j=1,2, \cdots, k)$. Whence

$$
b_{n+1} \leqq \frac{2}{r^{n}}+\frac{b_{n-1}}{r^{2}}+\frac{1}{r^{n+2}}\left(\frac{r^{n}}{p^{n}}-2+\frac{p^{n}}{r^{n}}\right) \sum_{j=1}^{k} m_{j}\left(\frac{r^{2}}{p_{j}^{2}}-1\right) .
$$


Using the inequality (4.2) and letting $r \rightarrow 1$ on a suitable sequence yields (4.22) $b_{n+1} \leqq 2+b_{n-1}+\left(\frac{1}{p^{n}}-2+p^{n}\right)=b_{n-1}+p^{n}+\frac{1}{p^{n}}, \quad n=1,2, \cdots$.

Since $b_{0}=0$ and $b_{1}=1$ an easy induction with (4.22) gives the right side of (4.17) and (4.18). Moreover, if $f(z) \in \mathrm{TM}(p)$, so also is $-f(-z)$. Clearly, this gives the left side of (4.17), and if $F(z)$ is an extremal function for the right side of (4.17) then $-F(-z)$ is an extremal function for the left side. These bounds are sharp, for if $2 s=p+p^{-1}$ in (2.3) then for $F_{s}(z), b_{n}=B(n, p)$, for $n=2,3, \cdots$.

To obtain the lower bound in (4.18) consider along with the non-negative integral (4.13) the obvious inequality

$$
\int_{0}^{2 \pi} v(r, \theta) \sin \theta \frac{\sin n \theta}{\sin \theta} d \theta \geqq \mu(n) \int_{0}^{2 \pi} v(r, \theta) \sin \theta d \theta
$$

where $\left|p_{k}\right|<r<\left|p_{k+1}\right|$. This yields $b_{n}^{(k)} r^{n}-b_{-n}^{(\boldsymbol{k})} r^{-n} \geqq \mu(n)\left(b_{1}^{(\boldsymbol{k})} r-b_{-1}^{(\boldsymbol{k})} r^{-1}\right)$, for $n=2,3, \cdots$, and using (4.12) this becomes

$$
b_{n} \geqq \frac{\mu(n)}{r^{n+1}}\left(r^{2}-\sum_{j=1}^{k} m_{j}\left(\frac{r^{2}}{p_{j}^{2}}-1\right)\right)+\frac{1}{r^{n}} \sum_{j=1}^{k} \frac{m_{j}}{p_{j}}\left(\frac{r^{n}}{p_{j}^{n}}-\frac{p_{j}^{n}}{r^{n}}\right) .
$$

Clearly, when $n$ is odd the last sum in (4.24) is positive and may be dropped. Further, $\mu(n)<0$, so the first sum may also be dropped, giving $b_{n} \geqq \mu(n) r^{1-n}$. Letting $r \rightarrow 1$ on a suitable sequence yields the desired lower bound. Clearly the equality sign can hold only if the function has no poles in $E$. Thus the extremal function here coincides with that found in [15], namely $F_{s}(z)$, with $s=\cos \theta_{0}$, where $\theta=\theta_{0}$ yields the minimum value $\mu(n)$ in (4.16). Of course $\theta_{0}$ and $F_{s}(z)$ depend on the index $n$.

TheOREM $7^{*}$. Let $T M^{*}(\beta)$ be the subclass of $T M^{*}$ of functions for which the nonzero poles $p_{j}$ satisfy $\left|p_{j}\right| \geqq p>0, j=1,2, \cdots$. If $\phi(z) \in T M^{*}(p)$ has the form (1.3) in a neighborhood of $z=0$, then $\beta_{1} \geqq-1$ and for $n=2,3, \cdots$

$$
-\left(1+\beta_{1}\right) B(n, p) \leqq \beta_{n} \leqq\left(1+\beta_{1}\right) B(n, p),
$$

$n$ even,

and

$$
\left(1+\beta_{1}\right) \mu(n) \leqq \beta_{n} \leqq\left(1+\beta_{1}\right) B(n, p),
$$

$n$ odd,

and all these bounds are sharp.

The proof closely parallels that of Theorem 7 so we merely outline it, indicating the altered equations by affixing ${ }^{*}$ to the original number. As before, the poles $p_{j}$ are numbered in order of increasing absolute value, but omitting the pole at $z=0$. Then 


$$
\beta_{n}^{(k)}=\beta_{n}-\sum_{j=1}^{k} \frac{m_{i}}{p_{j}^{n+1}}, \quad n=0, \pm 1, \pm 2, \cdots
$$

where $\beta_{-1}=1$ and $\beta_{n}=0$ for $n<-1$. The integral (4.13) yields

$$
\sum_{j=1}^{k} m_{j}\left(\frac{r^{2}}{p_{j}^{2}}-1\right) \leqq 1+r^{2} \beta_{1}
$$

and hence as $r \rightarrow 1$ on a suitable sequence

$$
\sum_{j=1}^{\infty} m_{j}\left(\frac{1}{p_{j}^{2}}-1\right) \leqq 1+\beta_{1}
$$

and hence $\beta_{1} \geqq-1$.

The integral (4.19) now leads to

$$
\begin{aligned}
\beta_{n+1} \leqq & \frac{2}{r^{n+2}}\left(\beta_{1} r^{2}+1\right)+\frac{\beta_{n-1}}{r^{2}}-\frac{\beta_{-n+1}}{r^{2 n}} \\
& +\frac{1}{r^{n+2}}\left(\frac{r^{n}}{p^{n}}-2+\frac{p^{n}}{r^{n}}\right) \sum_{j=1}^{k} m_{j}\left(\frac{r^{2}}{p_{j}^{2}}-1\right) .
\end{aligned}
$$

Using $\left(4.2^{*}\right)$ and letting $r \rightarrow 1$ gives

$$
\beta_{n+1} \leqq \beta_{n-1}-\beta_{-n+1}+\left(1+\beta_{1}\right)\left(p^{n}+\frac{1}{p^{n}}\right), \quad n=1,2, \cdots,
$$

and induction on $n$ yields the upper bound in $\left(4.17^{*}\right)$ and $\left(4.18^{*}\right)$. The lower bound in $\left(4.17^{*}\right)$ is trivial. The integral (4.23) gives

$\left(4.24^{*}\right) \quad \beta_{n} \geqq \frac{\mu(n)}{r^{n+1}}\left[\beta_{1} r^{2}+1-\sum_{j=1}^{k} m_{j}\left(\frac{r^{2}}{p_{j}^{2}}-1\right)\right]+\frac{1}{r^{n}} \sum_{j=1}^{k} \frac{m_{j}}{p_{j}}\left(\frac{r^{n}}{p_{j}^{n}}-\frac{p_{j}^{n}}{r^{n}}\right)$,

for $n=2,3, \cdots$. As in the proof of Theorem 7 , this gives the lower bound in $\left(4.18^{*}\right)$.

We show that these bounds are sharp by exhibiting the extremal functions. With $p$ fixed, $0<p<1$, and $\beta_{1}>-1$, set $2 s=p+p^{-1}$. Then the function

$$
\begin{aligned}
\phi_{\varepsilon}(z) & =-\frac{1}{z}+\beta_{0}-z+\left(1+\beta_{1}\right) \frac{z}{1-2 s z+z^{2}} \\
& =-\frac{1}{z}+\beta_{0}+\beta_{1} z+\sum_{n=2}^{\infty}\left(1+\beta_{1}\right) B(n, p) z^{n}
\end{aligned}
$$

belongs to $\mathrm{TM}^{*}(p)$ and the upper bound in $\left(4.17^{*}\right)$ and $\left(4.18^{*}\right)$ is attained for each $n>1$. The lower bound in $\left(4.17^{*}\right)$ is attained for the function $-\phi_{s}(-z)$. Finally consider the function 


$$
\begin{aligned}
\phi(z) & =-\frac{1}{z}+\beta_{0}-z+\left(1+\beta_{1}\right) \frac{z}{1-2 z \cos \theta_{0}+z^{2}} \\
& =-\frac{1}{z}+\beta_{0}+\beta_{1} z+\sum_{n=2}^{\infty}\left(1+\beta_{1}\right) \frac{\sin n \theta_{0}}{\sin \theta_{0}} z^{n} .
\end{aligned}
$$

Here again $\phi(z) \in \mathrm{TM}^{*}(p)$, and if $\theta_{0}$ is chosen properly (different for each index $n$ ) then for this function the lower bound in $\left(4.18^{*}\right)$ is attained.

As a corollary we have that if $\phi(z) \in \mathrm{TM}^{*}$ and if $\beta_{1}=-1$, then $\phi(z) \equiv-z$ $+\beta_{0}-z^{-1}$. Theorem $7^{*}$ contains as a special case a theorem due to Gel'fer [4], who considered typically-real functions with only a single pole located at the origin.

THEOREM 8. Let $f(z) \in T M(p)$ have the form (1.1), then

$$
-1 \leqq b_{3}-b_{2}^{2} \leqq p^{2}+1+p^{-2} \equiv B(3, p)
$$

and these bounds are sharp.

Proof. Since the coefficients are real, $b_{3}-b_{2}^{2} \leqq b_{3} \leqq B(3, p)$ by Theorem 7 . If $2 s=p+p^{-1}$ then $\left(F_{s}(z)-F_{s}(-z)\right) / 2 \in \mathrm{TM}(p)$ and shows that the upper bound in (4.25) cannot be decreased. For the lower bound recall that if $f(z) \in \mathrm{TM}$, then so also is

$$
f(z) /(1-c f(z))=z+\left(b_{2}+c\right) z^{2}+\left(b_{3}+2 b_{2} c+c^{2}\right) z^{3}+\cdots
$$

for any real $c$. From Theorem $7, b_{3}+2 b_{2} c+c^{2} \geqq \mu(3)=-1$. Taking $c=-b_{2}$ in (4.26) then gives the left side of (4.25). Since

$$
F_{s}(z)=\frac{z}{1-2 s z+z^{2}}=z+2 s z^{2}+\left(4 s^{2}-1\right) z^{3}+\cdots
$$

and since $b_{3}-b_{2}^{2}=4 s^{2}-1-(2 s)^{2}=-1$, the lower bound cannot be increased. The coefficients for functions in $\operatorname{TM}(p)$ are unbounded as $p \rightarrow 0$, yet the lower bound in (4.25) is independent of $p$, just as in one part of Theorem 7. This lower bound could also be proved by considering $\phi(z)=-f(z)^{-1}$ and applying the condition $\beta_{1} \geqq-1$ of Theorem $7 *$.

5. A representation theorem. We now show that any function in TM (or $\mathrm{TM}^{*}$ ) can be represented by a slight modification of a suitable $t(z)$ in TR (regular in $E$ ). The proof follows closely one used in a similar situation due to Schiffer and Bargemann, as presented by Wigner [16]. The precise results are as follows.

Theorem 9. Let $f(z) \in T M$. Then in $E$

$$
f(z)=\mu t(z)+\sum m_{j}\left(p_{j}^{-2}-1\right) \frac{z}{1-\left(p_{j}+p_{j}^{-1}\right) z+z^{2}},
$$


where $\mu \geqq 0, t(z) \in T R$, the sum extends over all poles $p_{j}$ of $f(z)$ in $E$, and $-m_{j}<0$ is the residüe of $f(z)$ at $p_{j}$. Further

$$
\mu+\sum m_{j}\left(p_{j}^{-2}-1\right)=1 .
$$

Theorem 10. Let $\phi(z) \in T M^{*}$. Then in $E$

$$
\phi(z)=-z^{-1}+\beta_{0}-z+\mu t(z)+\sum m_{i}\left(p_{j}^{-2}-1\right) \frac{z}{1-\left(p_{j}+p_{j}^{-1}\right) z+z^{2}}
$$

(with the same notations as in Theorem 9). Further

$$
\mu+\sum m_{j}\left(p_{i}^{-2}-1\right)=1+\beta_{1} .
$$

Proof. Let us suppose (dropping subscripts) that $f(z)$ has a pole $p \neq 0$ with residue $-m<0$. Then with $2 s=p^{-1}+p, a(z) \equiv m\left(p^{-2}-1\right) F_{s}(z)$ has the same pole $p$ with the same residue $-m$, so that $g(z) \equiv f(z)-a(z)$ has in $E$ the same poles as $f(z)$, except that at $z=p, g(z)$ is regular. Further, except for the poles, $g(z)$ is real on the real axis. We shall show that $g(z)$ satisfies the condition $\Im g(z) \Im z \geqq 0$ at the regular points in $E$, so that either $g(z) \equiv 0$, or $g(z) / g^{\prime}(0)$ $\in T M$. Now $a(z)$ is regular in the entire plane, except for the poles at $z=p<1$ and $z=1 / p$, and hence is uniformly continuous in any sufficiently narrow ring domain containing $|z|=1$. Further on $|z|=1, a(z)$ is real. Thus for any $\epsilon>0$, there is an $r<1$ such that for $r \leqq|z|<1,|\Im a(z)|<\epsilon / 2$. Suppose now that for a certain $z_{0}$ in $E, \Im g\left(z_{0}\right)=-\epsilon<0$, where $\Im z_{0}>0$. Consider $g(z)$ on the simple closed curve $\Gamma$ consisting of a semicircle $C:|z|=r$ in the upper halfplane, a set of disjoint semicircles $C_{j}$ with centers at the poles $p_{j} \neq p$ of $f(z)$, passing above the poles, and appropriate segments of the real axis joining the end points of $C_{j}$ and $C$ to form a simple closed curve (see Wigner [16, p. 41].) On the real axis $\Im g(z)=0$, except at the poles. In a neighborhood of $z=p_{j} \neq p$, $a(z)$ is regular so that the radius of $C_{j}$ can be taken so small that $|\Im a(z)|<\epsilon / 2$ and hence $\Im g(z)>-\epsilon / 2$ on $C_{j}$. Similarly on $C$. Hence a simple closed path $\Gamma$ can be found containing $z_{0}$ on the interior, on which $\Im g(z)>-\epsilon / 2$. Since $g(z)$ is regular inside and on $\Gamma$ this contradicts the assumption that $\Im g\left(z_{0}\right)=-\epsilon$. Now $g(0)=0$. If further $g^{\prime}(0)=0$ then $g(z) \equiv 0$. Otherwise $g^{\prime}(0)=1-m\left(p^{-2}-1\right)$ $>0$ and hence $g(z) / g^{\prime}(0) \in$ TM. It is now clear, by iteration, that for any finite set of poles the function

$$
g_{k}(z)=f(z)-\sum_{j=1}^{k} m_{j}\left(p_{j}^{-2}-1\right) F_{s_{j}}(z)
$$

satisfies the condition (1.2) in $E$, and hence is either identically zero or

$$
g_{k}^{\prime}(0)=1-\sum_{j=1}^{k} m_{j}\left(p_{j}^{-2}-1\right)>0 .
$$

If $f(z)$ has only a finite number of poles the proof of Theorem 9 is complete. 
If $f(z)$ has infinitely many poles, then as a consequence of the inequality (5.6) (or (4.3)), the sequence $g_{k}(z)$ converges uniformly on any closed domain of $E$, not containing a pole of $f(z)$. The limit function $g(z)$ satisfies the condition (1.2) and has no poles in $E$. It is either identically zero or $g^{\prime}(0)=\mu>0$, and $g(z)=\mu t(z)$ where $t(z) \in \mathrm{TR}$, and $\mu$ satisfies equation (5.2).

Theorem 10 is now an easy consequence of Theorem 9 , for if $\phi(z) \in \mathrm{TM}^{*}$, then by an argument similar to the one just given $f^{*}(z) \equiv \phi(z)-\beta_{0}+z+z^{-1}$ satisfies the condition (1.2) and hence is either identically zero, or

$$
f^{*}(z) /\left(1+\beta_{1}\right)=f(z) \in \mathrm{TM} .
$$

Herglotz [7] gave a representation as a Stieltjes integral for functions with positive real part in $E$. On the basis of this representation, Robertson [12] pointed out that if $f(z) \in \mathrm{TR}$ then there is a nondecreasing function $\alpha(\theta)$ such that

$$
f(z)=\frac{1}{\pi} \int_{0}^{\pi} \frac{z d \alpha(\theta)}{1-2 z \cos \theta+z^{2}}
$$

and he applied this formula in [14]. It was rediscovered by Goluzin [5] and generalized by $\mathrm{Li}[10]$ to functions typically-real and regular in circular rings.

On the basis of (5.1) and (5.7) it is easy to formulate a Stieltjes integral representation for the functions in the class TM. We refrain from pursuing the details, but from such a representation a large variety of results would flow. To mention just a few, this would give new proofs of our Theorems 2, 3, 4 , and 7 , and in addition would give immediately the precise domain of variability for the derivatives $f^{(k)}(z)$, for $f(z)$ in TM.

6. A remark on univalent functions. Let $U(p)$ denote the class of functions of the form (1.1) univalent in $E$, with pole at $z=p$. Since the extremal functions in Theorem 7 are also univalent, this theorem solves simultaneously the problem of determining the sharp bound for $b_{n}$ for functions in $U(p)$ in the special case that all the coefficients are real. It is natural to conjecture that for any $f(z) \in U(p),\left|b_{n}\right| \leqq B(n, p)$, a conjecture which contains the one $\left|b_{n}\right| \leqq n$ for regular univalent functions as a special case. In this direction Komatu and Ladegast have proved independently that if $f(z) \in U(p)$ then $\left|b_{2}\right| \leqq p^{-1}+p,[8$, p. 272] and $[9$, p. 134]

\section{REFERENCES}

1. C. Carathéodory, Über den Variabilitätsbereich der Koeffizienten von Potenzreihen, die gegebene Werte nicht annehmen, Math. Ann. vol. 64 (1907) pp. 95-115.

2. - Über den Variabilitätsbereich der Fourier'schen Konstanten von positiven harmonischen Funktionen, Rend. Circ. Mat. Palermo vol. 32 (1911) pp. 193-217.

3. L. R. Ford, Automorphic functions, New York, McGraw-Hill, 1929. 
4. S. A. Gel'fer, On the coefficients of typically-real functions, Doklady Akad. Nauk. S.S.S.R. vol. 94 (1954) pp. 373-376.

5. G. M. Goluzin, On typically-real functions, Rec. Math. (Mat. Sbornik) N.S. vol. 27 (69) (1950) pp. 201-218.

6. A. W. Goodman and M. S. Robertson, A class of multivalent functions, Trans. Amer. Math. Soc. vol. 70 (1951) pp. 127-136.

7. G. Herglotz, Über Potenzreihen mit positivem, reellen Teil im Einheitskreis, Ber. Verh. Sächs. Akad. Wiss., Leipzig, 1911, pp. 501-511.

8. Y. Komatu, Note on the theory of conformal representation by meromorphic functions I and II, Proc. Imp. Acad. Tokyo vol. 21 (1945) pp. 269-277 and 278-284.

9. K. Ladegast, Beiträge zur Theorie der schlichten Funktionen, Math. Zeit. vol. 58 (1953) pp. 115-159.

10. E. P. Li, On typically-real functions in a circular ring, Doklady Akad. Nauk S.S.S.R. vol. 92 (1953) pp. 699-702.

11. Z. Nehari and B. Schwarz, On the coefficients of univalent Laurent series, Proc. Amer. Math. Soc. vol. 5 (1954) pp. 212-217.

12. M. S. Robertson, On the coefficients of a typically-real function, Bull. Amer. Math. Soc. vol. 41 (1935) pp. 565-572.

13. - The variation of the sign of $V$ for an analytic function $U+i V$, Duke Math. $J$. vol. 5 (1939) pp. 512-519.

14. - On the coefficients of univalent functions, Bull. Amer. Math. Soc. vol. 51 (1945) pp. 733-738.

15. W. Rogosinski, Über positive harmonische Entwicklungen und typisch-reelle Potenzreihen, Math. Zeit. vol. 35 (1932) pp. 93-121.

16. E. P. Wigner, On a class of analytic functions from the quantum theory of collisions, Ann. of Math. vol. 53 (1951) pp. 36-67.

The University OF KentuCKy,

LEXington, Ky. 